\title{
Reflexiones sobre la ética de la investigación en Colombia ${ }^{i}$ Reflections on Ethics of Research in Colombia
}

\author{
Por:Hugo Nelson Castañeda Ruiz, ${ }^{1}$ Ángela María Cómez Osorio² \& Ángela María Londoño Jaramillo
}

1. Abogado Universidad de San Buenaventura Medellín. Especialista en Responsabilidad Civil, Universidad Autónoma Latinoamericana, Medellín. Magíster (c) en Derecho y Economía, Universidad de Buenos Aires, Argentina. Docente del CIDEH, Universidad de San Buenaventura Medellín. Integrante del Comité de Bioética Institucional e investigador del Grupo Interdisciplinario para el Desarrollo del Pensamiento y la Acción Dialógica (CIDPAD), Orcid: https://0rcid.org/0000-0002-6324-8201 Scholar: https://scholar.google.es/citations?user=bdrNjtoAAAA]\&hl=es. Contacto: hugo.castaneda@usbmed.edu.co

2. Socióloga Universidad de San Buenaventura Medellín. Especialista en Gestión de la Calidad Universitaria, Universidad de San Buenaventura Medellín. Docente del CIDEH Universidad de San Buenaventura Medellín. Investigadora del Crupo Interdisciplinario para el Desarrollo del Pensamiento y la Acción Dialógica (CIDPAD). Orcid: https:// orcid.org/0000-0003-1301-0980Scholar: https://scholar.google.es/citations?user=hqgOd5gAAAA]\&hl=es Contacto: angela.gomez@usbmed.edu.co

3. Comunicadora social y periodista de la Universidad de Manizales. Magíster en educación y desarrollo humano de la Universidad de Manizales. Docente del CIDEH, Universidad de San Buenaventura Medellín. Investigadora del Grupo Interdisciplinario para el Desarrollo del Pensamiento y la Acción Dialógica (CIDPAD). Orcid: https:// orcid.org/0000-0001-7390-1801 Scholar: https://scholar.google.com/citations?user=5/snWpMAAAAJ\&hl=es Contacto: angela.londono@usbmed.edu.co

\section{G OPEN ACCESS}

(c) $\underset{\mathrm{BY}}{\mathrm{B} \text { No } \mathrm{NC}}$

Copyright: $\odot 2020$ El Ágora USB.

La Revista El Ágora USB proporciona acceso abierto a todos sus contenidos bajo los términos de la licencia creative commons Atribución-NoComercial-SinDerivar 4.0 Internacional (CC BY-NC-ND 4.0)

Tipo de artículo: Reflexión Recibido: enero de 2020

Revisado: febrero de 2020

Aceptado: mayo de 2020

Doi: $10.21500 / 16578031.5144$

Citar así: Castañeda Ruiz, H., Cómez Osorio, Á. \& Londoño Jaramillo, Á. (2020). LReflexiones sobre la ética de la investigación en Colombia. El Ágora USB, 2O(2). 283-297

Doi: $10.21500 / 16578031.5144$

\section{Resumen}

Se reflexiona en torno al diseño normativo que regula la investigación en Colombia y las prácticas de los investigadores desde un enfoque ético, abordando asuntos problemáticos en las ciencias de la salud, las ciencias sociales y las disciplinas ambientales, para concluir que se necesita fortalecer el control del Estado en estos temas, la actualización de la normatividad y una mayor formación de los investigadores y miembros de Comités de Ética en investigación, especialmente en: la protección de los participantes, el cuidado de los animales, los conflictos de interés, el manejo de comunidades y de grupos vulnerables, los protocolos comunitarios, el consentimiento informado, la consulta previa, el cuidado de la biodiversidad y el acceso a recursos genéticos.

Palabras clave: ética de la investigación; grupos vulnerables; conflicto de interés; biodiversidad; acceso a recursos genéticos.

\section{Abstract}

This paper reflects on the normative design, which regulates research in Colombia and the practices of researchers from an ethical approach, by addressing critical issues in the health sciences, social sciences, and environmental disciplines, to conclude that it is necessary to strengthen state control in these issues, the updating of the regulations and greater training of researchers and members of Ethics Committees in research, especially in the protection of participants, animal care, conflicts of interest, the management of communities, and vulnerable groups, community protocols, informed consent, prior consultation, biodiversity care, and access to genetic resources.

Keywords: Ethics of Research; Vulnerable Groups; Conflict of Interest; Biodiversity; and Access to Genetic Resources. 


\section{Introducción}

Amira Abultaif Kadamani, hablando sobre la ética en la investigación del país dice: "Si la ciencia logra consolidarse como la locomotora de la innovación y el progreso nacional, la ética deberá ser su maquinista" (2018). Y es que, en los planteamientos propios del ámbito científico, la creación y divulgación de conocimientos deben contribuir al bienestar social, al crecimiento económico y a la conservación natural; sin embargo, no siempre el impacto ha sido positivo en la transformación de las condiciones de la calidad de vida de las personas, de las comunidades, o del medio ambiente. En algunos casos, los avances científicos han sido enfocados en contra de la supervivencia humana y de la cohesión social, así como también se han presentado conflictos territoriales y pérdidas en recursos naturales y biodiversidad.

Es por esto que se hace indispensable contar con la ética y la integridad científica como factores que generen confianza sobre los procesos científicos por parte de las instituciones y la sociedad en general; para ello es necesario que las investigaciones se realicen con rigor, esto es, que se utilicen métodos y técnicas sustentadas académicamente y que permitan confrontar los hallazgos o reproducirlos siguiendo los mismos procedimientos, que cumplan con las pautas y normas definidas en las leyes y políticas nacionales y ante todo, que se actúe con respeto y responsabilidad por el bien de los participantes, las poblaciones y los territorios intervenidos.

En la actualidad, los lineamientos éticos no son muy claros con los contextos de las diferentes ciencias o ámbitos disciplinares que producen el conocimiento, ni con las praxis. Ante lo cual es indispensable incorporar la dimensión de la responsabilidad como parte esencial en la formación de los investigadores, por esto se hace preciso que el sujeto investigador sea consciente de su labor y de la responsabilidad que esto implica, alejándose de las tendencias actuales, de ahí que se requiera, de acuerdo con Hans Jonás, que las personas actúen de manera que las consecuencias de sus acciones permitan la permanencia de la humanidad en el planeta, así como su diversidad cultural (Siqueira \& Eduardo, 2001, pp.279-280).

En Colombia, el principal ente reguladory promotor de las actividades de la ciencia, el desarrollo tecnológico y la innovación (ACTI) es el Ministerio de Ciencia, Tecnología e Innovación-Mincienciasanteriormente denominado Colciencias, en ese entonces conocido como el Departamento Administrativo de Ciencia, Tecnología e Innovación - Colciencias. El cual en los últimos años ha venido liderando la implementación de las políticas de ética en la investigación, bioética e integridad científica, que pretende la transformación de los científicos y académicos del país en torno a sus prácticas investigativas y divulgativas, tanto en el ámbito público como privado. Sin embargo, existe una gran distancia entre la praxis de la CT\&I, la normatividad en Colombia y los entes garantes de la ética en la investigación; razón por la cual se configura el siguiente artículo, el cual busca presentar una reflexión sobre las políticas, normatividad y la praxis actual en el campo de la ciencia, la tecnología y la innovación en Colombia, específicamente frente a los aspectos morales y éticos.

Para lograr lo anterior, este artículo aborda las siguientes temáticas: en la primera se describirán los lineamientos jurídicos que rigen la práctica investigativa en el país, en la segunda se presentará las principales críticas a la normatividad, luego, en una tercera parte, se hará una crítica a algunas prácticas científicas y divulgativas que se realizan por algunos académicos en el país, en cuarto lugar se analizan las políticas como respuesta a esas falencias jurídicas y sociales en los procesos de investigación, en la quinta parte se propondrán algunas alternativas de solución a las problemáticas señaladas y por último, se presentarán las conclusiones del texto. 


\section{Normatividad que regula aspectos éticos o disciplinarios de la} investigación en Colombia

Aunque no se puede confundir la ética con el derecho, se debe indicar que este último, sirve como referente para definir las responsabilidades y los alcances mínimos de la investigación científica, esto es, los criterios morales y jurídicos básicos que se deben tener en una investigación. En cambio, desde la ética se deberá reflexionar y deliberar en cada caso, para poder tomar decisiones correctas que aporten a la ciencia pero que, a su vez, respete los derechos de las personas participantes en los procesos investigativos, que se tengan criterios de cuidado con los animales que se encuentran en bioterios o que vayan a ser utilizados en investigación, o medidas de prevención y precaución en proyectos que puedan generar actividades que potencialmente presenten riesgo para seres humanos, animales o el medio ambiente.

Es de recordar que si bien la ciencia es necesaria para el desarrollo y el bienestar de las sociedades, también se debe reconocer que ha tenido usos que van en contravía de estas premisas, de ahí que se necesite un puente que conecte a la investigación y en general a la ciencia, con un sin fin de especialidades éticas, que permitan la protección del medio ambiente, el bienestar personal y comunitario y la supervivencia humana (Potter, 1999, p.40).

Se debe indicar que, en el sistema normativo y en la reflexión éticas sobre las prácticas y fines moralmente aceptables en la investigación científica, se ha tomado como referente a las ciencias de la salud y su historia es reciente, pues está ligada a la evolución contemporánea de los derechos humano.

Esto se hace evidente después de la segunda guerra mundial, donde muchos profesionales de la medicina, iniciaron investigaciones en nombre de la ciencia, favoreciendo el exterminio de pueblos, tanto fue así que, una vez terminada la guerra, y con los estudios psicológicos y psiquiátricos se determinó la existencia de un "síndrome de los campos de concentración" (Riquel me, 2004, p. 131) que padecieron muchos de los sobrevivientes del holocausto, esto debido a los horrores que vivieron personas internadas en estos lugares por los abusos físicos y psicológicos por parte del personal que se encontraba en estos sitios, así como, por las investigaciones denigrantes que atentaron contra la dignidad y que utilizaron a personas como cobayas humanas.

En el año de 1946, como consecuencia de lo ocurrido en nombre de la ciencia durante la segunda guerra mundial, se juzgó a los científicos nazis y japoneses bajo los cargos de crímenes contra la humanidad, y uno de los productos de estos juicios, fue la expedición del Código de Nuremberg (Tribunal Internacional de Nüremberg, 1946), cuyo fin fue reglamentar los procesos de investigación en las áreas biomédicas para que no se vuelvan a repetir las acciones del holocausto.

De igual forma, la Asociación Médica Mundial, aprobó en el año de 1964, en su 18a Asamblea, la Declaración de Helsinki sobre los Principios Éticos para las Investigaciones médicas con Seres Humanos (Asociación Médica Mundial, 2013), estas son recomendaciones que tienen pretensión de universalidad, para que los profesionales de la salud a nivel mundial, no repitan lo ocurrido en el Holocausto, con las investigaciones en esta área.

En el año 1979 se aprobó en Estados Unidos de Norte América el Informe Belmont, instrumento que creó unos principios de ética biomédica (justicia, beneficencia -no maleficencia-y autonomía) como respuesta en este país a lo ocurrido con la población de Tuskegee, en el Estado de Alabama, donde se hicieron experimentos con seres humanos para comprender la evolución y tratamiento de la sífilis. Estos experimentos tuvieron problemas éticos como la segregación racial (se utilizó población afrodescendiente), falsos consentimientos informados, no preservar la integridad y la salud de los pacientes. Este estudio comenzó en el año 1932 y fue dirigido por el Dr. Taliaferro Clark, se utilizaron 400 personas enfermas y 200 sanas como grupo control. La población fue engañada para que participara del experimento. Este estudio se demoró casi 40 años y nunca se les suministró penicilina a los pacientes, a pesar de haber demostrado que ésta era eficiente para contrarrestar la 
enfermedad. Al terminar la investigación, 74 personas quedaron vivas, pero otros no contaron con la misma suerte, pues algunos fallecieron de la enfermedad, otros fueron contagiados e incluso 19 menores nacieron con sífilis (Osío Uribe, 2009).

En el año de 1982 aparece la primera versión de las "Pautas Éticas Internacionales para la Investigación Relacionada con la Salud con Seres Humanos", elaborada por el Consejo de Organizaciones Internacionales de las Ciencias Médicas (CIOMS) en colaboración con la Organización Mundial de la Salud (OMS), también conocida como las Pautas CIOMS, las cuales complementaron y en algunas pocas cosas suavizaron las exigencias de la Declaración de Helsinki. La idea fue contar con unas pautas éticas más detalladas que se deberían tener en cuenta en los procesos de investigación.

Bajo esta variada influencia, en Colombia, desde las ciencias de la salud se creó hace 26 años, la Resolución 8430 de 1993 del Ministerio de Salud, que es la principal norma sobre investigación en salud y que se erige como el referente nacional sobre ética en la investigación científica en casi todas las disciplinas. Tal es así que en las diferentes Universidades e instituciones que cuentan con Comités de ética en la investigación o bioética, citan como referente esta norma. Dentro de las bondades de esta norma se encuentra la obligatoriedad de solicitar el consentimiento al participante, previa información sobre las consecuencias y riesgos, la protección a grupos vulnerables, el mayor cuidado que se debe tener en investigaciones con riesgo mayor al mínimo, entre otros.

No se pueden desconocer los derechos, libertades y principios constitucionales que reglamentan y fundamentan el tema, como el derecho a la salud, el derecho a la vida, la dignidad humana, la prohibición de tratos crueles y degradantes, el derecho a la igualdad, el derecho a la intimidad personal y familiar, etc.

En el caso de la investigación con medicamentos aplicados a seres humanos, la principal norma en Colombia es la Resolución 2378 de 2008 del Ministerio de Protección Social (Resolución 2378: Las Buenas Prácticas Clínicas para las instituciones que conducen investigación con medicamentos en seres humanos, 2008) (hoy Ministerio de Salud), en el que se adoptan la guía de Buenas Prácticas Clínicas (BPC) bajo el amparo de la Organización Panamericana de la Salud (OPS), cuya crítica es que fue un programa patrocinado por la industria farmacéutica, que estandarizó y optimizó el proceso de análisis que puede perjudicar la reflexión ética (Obando, 2015, p. 184).

Aparte de las normas ya mencionadas que son del ámbito clínico en su mayoría, pero que se ha ido extrapolando a otros ámbitos, también se debe tener en cuenta otras normas, en algunos casos, menos conocidas, y son las siguientes:

A la propiedad intelectual como los derechos de autor, ley 23 de 1982 (Ley número 23: Sobre los Derechos de Autor, 1982), Derechos de propiedad industrial, ley 178 de 1994 y Decisión Andina 486 de 2000 (Decisión 486: Régimen Común sobre Propiedad Industrial, 2000), Derechos de obtentor, Decisión Andina 345 de 1993 y Ley 243 de 1995(Decisión 345: Régimen Común de Protección a los Derechos de los Obtentores de Variedades Vegetales, 1993),

a la protección de los animales (Ley 84: Estatuto Nacional de Protección de los Animales, 1989), aunque en el momento en que se escribió este texto, en el Congreso de la República se encuentra en trámite el proyecto de ley 266C-2019 que podría modificar la ley 84.

Y en temas de biodiversidad, especialmente en recolección de especies y acceso al recurso genético: la decisión andina 391 sobre datos genéticos, la ley 165 de 1994 (Decisión 391: Régimen Común sobre Acceso a los Recursos Cenéticos, 1996) que aprueba el Convenio sobre diversidad biológica, el decreto 309 de 2000 que reglamenta la investigación científica sobre diversidad biológica, el Decreto 1375 de 2013 que reglamenta las colecciones biológicas, el Decreto 1376 de 2013 sobre recolección de especímenes de especies silvestres, al igual que el decreto 3016 del mismo año, que reglamenta el permiso de Estudio para la recolección de especies, la ley 13 de 1990 que en el Título III Capítulo I, regula algunas cuestiones sobre la 
investigación en la actividad pesquera y le otorga funciones de autorización y vigilancia al Instituto Nacional de Pesca y Acuicultura (INPA), la resolución 1348 de 2014 del Ministerio del medio Ambiente que establece las actividades que configuran acceso a los recursos genéticos y sus productos derivados, la resolución 3492 de 1998 y la resolución 2935 de 2001 que tratan sobre las condiciones de bioseguridad por la producción, liberación y comercialización de Organismos Cenéticamente Modificados (OGM).

Entre los fines de la Decisión Andina 391 de 1996 se reconoce la contribución de las étnias y comunidades locales en la conservación y uso sostenible de la biodiversidad de sus territorios (Decisión 391: Régimen Común sobre Acceso a los Recursos Genéticos, 1996), se establecieron mecanismos para que los países, que son miembros de la Comunidad Andina de Naciones, desarrollen conocimientos sobre su diversidad biológica y se proteja el conocimiento que tienen las comunidades indígenas, afroamericanas y locales sobre sus recursos. Por eso, cuando se va a trabajar con biodiversidad es necesario pedir autorización a las autoridades ambientales y cuando implique recolección de especies en zonas donde existen comunidades (independientemente de su clasificación), se debería utilizar el mecanismo de la consulta previa, si se hace una interpretación amplia de esta Decisión Andina, armonizada con otras normas jurídicas, pero en la práctica, solo se hace la consulta previa con comunidades indígenas y afrodescendientes, de acuerdo con una interpretación restrictiva del decreto 1320 de 1998 (Decreto 1320: La consulta previa con las comunidades indígenas y negras para la explotación de los recursos naturales dentro de su territorio., 1998) y del artículo 25 del decreto 309 de 2000 (Decreto 309: Se reglamenta la investigación científica sobre diversidad biológica., 2000). En la Decisión Andina 391 de 1996 también se reglamentan los contratos de acceso a los recursos genéticos y productos derivados, definiéndolos como el "acuerdo entre la Autoridad Nacional Competente en representación de la Estado y una persona, el cual establece los términos y condiciones para el acceso a recursos genéticos, sus productos derivados y, de ser el caso, el componente intangible asociado". Tanto en la Decisión Andina como en la resolución 1348 de 2014 se presupone que la Nación es la dueña de la biodiversidad y, por esto, es el único facultado para otorgar permisos y establecer las condiciones de cómo se accederá y qué beneficios permitir a los investigadores. Por ejemplo, si un grupo de investigación quiere acceder a los recursos genéticos o a elaborar productos derivados de alguna especie, es necesario que la Institución a la cual pertenece dicho grupo, deba celebrar este tipo de contratos con el Estado, específicamente con la Dirección de Bosques, Biodiversidad y Servicios Ecosistémicos, que de acuerdo con el numeral 14 del artículo 16 del Decreto Ley 3570 de 2011, es la entidad del gobierno autorizada para ello.

Dentro de las críticas que se pueden hacer a estas prácticas es que frente a los conocimientos tradicionales y la diversidad biológica en territorios donde se encuentran comunidades locales campesinas, también debería existir la consulta previa, como mecanismo de protección, con todo lo que ello involucra, incluido el "Consentimiento libre, previo e informado", ya que existe la misma razón para el uso de esta figura que el que tienen las comunidades étnicas, pues los campesinos y comunidades locales han tenido relación con su territorio, lo conocen, han obtenido conocimientos tradicionales de esa interacción y si no se hace la consulta previa, serían comunidades con un mayor nivel de desprotección y de vulnerabilidad que los grupos étnicos. Igual sucede con los contratos de acceso a recursos genéticos y productos derivados, en los que se debe incluir la consulta previa y la devolución de beneficios, cuando la recolección se realice en territorios comunitarios, sea de grupos étnicos o de campesinos locales.

Colombia también debería considerar la aprobación del Protocolo de Nagoya, que puede servir de complemento a la Decisión Andina 391 de 1996, en la medida en que introduce los protocolos comunitarios, con los que las comunidades pueden establecer las reglas de juego, sin contradecir la constitución y las leyes nacionales, para que los investigadores e instituciones puedan acceder a 
las comunidades, reforzando la ética en los procesos de bioprospección y la protección frente a la biopiratería.

Para culminar este tema, es de resaltar el trabajo de Cómez y Nemogá, quienes hicieron un estudio sobre las solicitudes de Acceso al Recurso Cenético (ARG) y los Permisos de Estudio con Fines de Investigación Científica (PEFIC) ante el Ministerio del Medio Ambiente anterior al año 2006 y encontraron muchas dificultades por desistimiento o archivo de los procesos de solicitud, en una buena proporción de los 37 expedientes que se revisaron. Ellos adjudican dicha situación a los trámites engorrosos para obtener el permiso, desorganización en el manejo de la información y posiblemente, por desconocimiento de los investigadores sobre estos trámites, por ejemplo: en 7 investigaciones se indicaron la presencia de comunidades étnicas, pero solo 1 tenía adelantada la consulta previa. De estos resultados, los investigadores infieren que se pueden estar ejecutando investigaciones de este tipo sin el cumplimiento de estos requisitos, lo cual incentivaría la ilegalidad en estos procesos (Gómez \& Nemogá, 2007).

Aunque estos trámites se han mejorado desde el año 2006 hasta el presente y se está tratando de incentivar la investigación en éste campo, verbigracia con el proyecto Colombia BIO, es necesario fortalecer: a. la formación de los investigadores con relación a los trámites y a la ética en la investigación, b. los procesos de control de las investigaciones donde se requiera acceso al recurso genético, o el análisis de recursos biológicos, especialmente aquellas que se solicita la salida de muestras a organismos extranjeros, y c. dar una mayor publicidad a los proyectos en este campo, especialmente aquellos que son estratégicos para el interés nacional, con el fin de tener veedurías o ejecutar los mecanismos de control social por parte de la ciudadanía.

Dentro de los principios jurídicos utilizados por la mayoría de Comités de Ética en Investigación, es el de precaución, principio que tiene su origen en el derecho ambiental, teniendo como primer antecedente internacional de su utilización fue en la década de 1970 en Alemania, para hacer frente a los efectos nocivos de los productos químicos. Este principio también se encuentra consagrado en la Declaración de Río de Janeiro y en Colombia, fue introducido por la Ley 99 de 1993 y desarrollado en la amplia jurisprudencia de la Corte Constitucional de Colombia, desde la sentencia C-293 de 2002 (aunque existen antecedentes en las sentencias C-073 de 1995 y T-574 de 1996), con una variación con relación a la definición que aparece en la Declaración de Río de Janeiro, y que lo eleva a principio constitucional de aplicación excepcional por parte de los operadores jurídicos, definiéndolo como la potestad de actuar para impedir un daño grave e irreversible "para los recursos naturales o la salud humana" que pueda generar una obra o actividad, así no exista la certeza científica absoluta de la posible ocurrencia del daño. Este principio, de acuerdo con la Corte Constitucional, tiene los siguientes elementos:

"1. Que exista peligro de daño; 2. Que éste sea grave e irreversible; 3. Que exista un principio de certeza científica, así no sea ésta absoluta; 4. Que la decisión que la autoridad adopte esté encaminada a impedir la degradación del medio ambiente. 5. Que el acto en que se adopte la decisión sea motivado. (Sentencia C 293, 2002)"

Como ya se mencionó, el desarrollo del principio de precaución en lo jurídico, ha tenido un desarrollo exclusivo en el ámbito ambiental, no obstante, por los elementos que describe la Corte Constitucional, este principio es perfectamente aplicable al ámbito de la investigación científica, no necesariamente en investigaciones relacionadas con el medio ambientales, sino que el análisis excepcional se puede hacer también en aquellos ámbitos técnico-científicos que impliquen intervención o que puedan afectar la salud mental y física de seres humanos y animales. En éste momento, Minciencias (antes conocido como Colciencias) y un grupo de académicos de diferentes universidades y entidades del país, están implementando la política de Ética de la Investigación, Bioética e 
Integridad Científica, la cual se encuentra regulada en la resolución 314 de 2018. Este es un proyecto ambicioso e interesante donde se priorizaron tres variables independientes (formación, institucionalidad y gobernanza) que permitirán generar, en el mediano plazo, un cambio en las prácticas de la mayoría de los investigadores y de las entidades clave que financian la investigación en el país.

\section{Críticas a la práctica investigativa en Colombia}

Al realizar un análisis crítico a las políticas y a las prácticas investigativas en Colombia se observa que la falta de una visión clara sobre las funciones que debe cumplir la CT\&l en el desarrollo del país y la politización de los recursos destinados a este ámbito, ha llevado a una postura netamente instrumentalizada de la ciencia y ha generado prácticas académicas en universidades e instituciones que minimizan los cánones éticos para cumplir con los indicadores requeridos. Es por esto que en algunas ocasiones los aspectos éticos en los procesos de investigación se han convertido en un requisito de relleno, dejando a un lado la opción de reflexión y análisis acerca de la importancia de la ciencia conducida éticamente. (Suárez Obando, 2015). Por esta razón, se va a realizar un análisis identificando algunos elementos necesarios de ser reflexionados desde una ética de la investigación.

Para analizar las prácticas éticas en el sistema de investigación en nuestro país, se proponen como elementos de partida: los objetivos de los procesos, las prácticas, los actores y aquellos mecanismos de control que verifiquen y garanticen el adecuado funcionamiento de las investigaciones y, por lo tanto, el impacto que estas puedan tener en seres humanos, animales y ecosistemas.

Al observar los objetivos de la ciencia, de acuerdo a la Unesco, el servicio que ésta debe prestar a la humanidad es aportar a las personas una mejor comprensión del medio natural y las sociedades, mejorar la calidad de vida de los seres humanos y propender por un ambiente sano y sostenible para las generaciones que están porvenir. Asimismo, en Colombia de acuerdo a las políticas vigentes, la ciencia, la tecnología y la innovación deben direccionarse hacia el impulso del desarrollo económico y social del país, aportando a un bienestar y calidad de vida de las diferentes poblaciones (Conpes). Sin embargo, la gran paradoja es que el avance de la ciencia y la tecnología han generado problemas de sostenibilidad por los daños a la naturaleza y por los desequilibrios sociales que se producen, por ejemplo la brecha tecnológica y económica entre Norte y Sur (Unesco, 1999).

En nuestro país, de acuerdo al Observatorio de Ciencia y Tecnología, en el año 2018, la inversión nacional en Actividades de Ciencia, Tecnología e Innovación (Acti) por tipo de recurso tuvo la siguiente distribución: el sector público participó con 32,36\%, el sector privado con un $67,45 \%$, el sector internacional con un 0,19\% (Pardo Martínez \& Cotte Poveda, 2018, p. 30) y en cuanto a la inversión nacional en ACTI por tipo de entidad ejecutora, las empresas participan con un 51,94\%, las instituciones de educación superior con un $12,33 \%$, las entidades gubernamentales con un $22,30 \%$, los centros de investigación y desarrollo tecnológico con un 9,86\%, los hospitales y clínicas con un $1,44 \%$, las Instituciones Privadas sin Fines de Lucro al servicio de las empresas con un 1,64\% y las ONG's, asociaciones y agremiaciones profesionales con un 0,49\% (Pardo Martínez \& Cotte Poveda, 2018, p. 33). Esto evidencia que el aporte destinado a las ciencias en el país se direcciona, en su mayoría, a intereses particulares que se materializan en desarrollo tecnológico, comercial o en indicadores que ayuda a particulares y en muchos casos sin aportarle a las comunidades o a la sostenibilidad.

Este escenario se hace más evidente cuando se revisan los sistemas de calidad de la investigación, pues en los escenarios privados y educativos se han configurado sus sistemas de valoración en indicadores que son estructurados bajo los lineamientos de mecanismos internacionales de competitividad. Las empresas focalizan en sus áreas de I+D, aquellos adelantos necesarios para la generación de productos comercialmente competitivos que les permite posicionarse en el mercado e incrementar ingresos. En el caso de las Instituciones de Educación superior, estas están más focalizadas en incrementar indicadores de producción académica como aquellos estipulados desde Minciencias por los procesos de calidad de la educación, por ejemplo, en términos de publicación, si 
un investigador quiere generar un producto tipo TOP, los artículos de investigación deben ser categoría Art_A1 o Art_A2, esto es, artículos de investigación que se encuentren en el cuartil 1 o 2 de ISI, ahora conocido como Web of Science o WOS, o Scopus (Departamento Administrativo de Ciencia, Tecnología e Innovación - Colciencias, 2016), métricas que son elaboradas por entidades del sector privado y que privilegian en sus sistemas las publicaciones de repositorios privados, es decir que, para acceder a artículos bien posicionados en estas métricas, se debe pagar la suscripción a los repositorios, y si se quiere publicar en revistas de alto impacto, esto es, que aparecen en mejores posiciones en estos modelos de medición, se debe pagar sumas de dinero nada despreciables, para que se evalúe su pertinencia en la revista.

Lo anterior ha hecho que los investigadores estén más interesados en la generación de productos que sumen en sus currículos, que en concebir resultados necesarios para el desarrollo del país y la población. Específicamente en el área educativa, el foco ubicado en la generación de productos como artículos científicos, libros, patentes, software, prototipos, diseños industriales, nuevos materiales académicos, diseños curriculares, entre otros, ha conllevado prácticas que relegan los procesos metodológicos y de calidad a un segundo o tercer plano. En este escenario científico, Sánchez Vásquez manifiesta que los investigadores son "Un héroe que ha sufrido hoy día tantas transformaciones hasta convertirse en un "obrero sofisticado en el tiempo del post-capitalismo" (2018, p. 80), donde se vuelve difuso el soporte ético y se libera al experto científico del compromiso con la sociedad. En otras palabras, los esfuerzos se concentran en usar los resultados obtenidos en publicaciones de carácter científico privado, y en pocas oportunidades son divulgadas en los contextos reales que propicien intervenciones eficaces en las poblaciones afectadas.

Todo esto viene acompañado por un sistema jurídico desactualizado y sin cohesión, que puede generar interpretaciones contradictorias, por unas instituciones que de cierta forma son débiles a la hora de hacer cumplir las normas y por una escasa formación en ética, bioética e integridad científica de los investigadores, que no les permite sensibilizarse frente a su responsabilidad y, en muchos casos, los ha llevado a priorizar sus propios intereses por encima de los intereses de personas o comunidades. En esta postura utilitarista por parte de los investigadores "las consideraciones éticas de abordar científicamente al "otro" son escasas" (Sánchez Vázquez, 2018), las poblaciones, los animales o los ecosistemas son asumidos como "objetos de investigación" lo cual elimina cualquier responsabilidad con su bienestar.

Para Fernando Suárez Obando cualquier investigación científica, en su diseño metodológico tiene una dimensión ética, indistintamente del área de conocimiento, los investigadores deben tener presente: a. La importancia de hacer una adecuada recolección de la información, b. Establecer mecanismos de protección de los datos, especialmente aquellos que pueden ser sensibles para los seres humanos involucrados, c. Establecer la rigurosidad del método y la cientificidad del proceso investigativo que permita un auténtico conocimiento (citado por Abultaif Kadamani, 2018). Sin embargo, en muchos casos se observan saltos metodológicos que impiden la veracidad de los datos y el adecuado manejo de la población o los ecosistemas, como son: falta de procesos de triangulación por limitaciones de tiempo o presupuestales, la falta de acercamiento a los pobladores, lo cual afecta no solo los datos sino que transgrede los derechos de las comunidades, actividades que pueden amenazar de forma grave o irreversible a los ecosistemas, por ejemplo: el uso de químicos, recolección de muestras sin consideración de las especies, siembra o implantación de especies no nativas, entre otros.

En renglones anteriores se manifestó la debilidad en el sistema normativo; actualmente existen diversos protocolos éticos y legales que buscan orientar el accionar del investigador en el ámbito científico. Estos sistemas, en su mayoría, surgen en las áreas de la salud como la medicina; lo cual conlleva a que las demás áreas o disciplinas los tomen como punto de referencia y no se tengan en 
cuenta las características propias del área, lo que puede generar consecuencias negativas al no tener presente posibles situaciones que afecten el bienestar humano y natural.

Por ejemplo, en la actualidad, frente a las condiciones de la naturaleza, los gobiernos han ido desarrollando protocolos como el de Kioto que buscó enfrentar las consecuencias humanas que inciden en el proceso del cambio climático a través de la reducción de las emisiones de gases efecto invernadero, el cual fue propuesto bajo el amparo del Convención Marco de las Naciones Unidas sobre el Cambio Climático (CMNUCC), o el protocolo de Nagoya sobre acceso a los recursos genéticos y participación justa y equitativa en los beneficios que se deriven de su utilización al convenio sobre la diversidad biológica, el cual tiene como objetivo que las sociedades participen de manera equitativa y justa en los beneficios derivados del uso de la diversidad biológica y de los recursos genéticos (Organización de las Naciones Unidas, 2011).

Sin embargo, pese a su existencia, hay muchos procesos científicos y gubernamentales que no los tienen en cuenta para sus diseños metodológicos ni para su implementación. De acuerdo con Fernando Suárez-Obando, las normas y protocolos internacionales, así como las normas nacionales que regulan las conductas de los investigadores, son aplicadas de forma incontrovertible por parte de Comités de Ética en la Investigación -CEI-, por las distintas autoridades y por muchos investigadores (citado por Abultaif Kadamani, 2018), que asumen sus directrices como listas de chequeo, sin la deliberación suficiente sobre la pertinencia en su aplicación. De acuedo a lo discutido en los Diálogos Nacionales sobre Ética de la Investigación:

Las normas, las instancias y las reglas no están hechas por procesos democráticos, pero deben apuntar a ellos, teniendo en cuenta que la investigación es un bien público. En todo caso, el tema se debe asumir como un proceso de aprendizaje, pues es la práctica y la experiencia la que va configurándolo" (Duque Ortiz, 2015, p. 100).

Para garantizar una adecuada práctica ética en los procesos investigativos, se han creado como principales mecanismos de control, de acuerdo con la Resolución 8430 de 1993 del Ministerio de Salud y la Resolución 2378 de 2008 del Ministerio de la Protección Social, los comités de ética en la investigación (CEI) o sus equivalentes como: los comités de bioética, los comités institucionales para el cuidado y uso de animales de laboratorio, los comités de propiedad intelectual, los comités editoriales (en cuanto a los asuntos éticos en publicaciones científicas) y las comisiones de bioseguridad (en cuanto a la seguridad de los seres humanos, animales y medio ambiente por uso de agentes biológicos, por productos químicos o por la realización de actividades peligrosas en laboratorio).

Es de destacar que, en la actualidad, cuando se hace un proceso de investigación en el país a nivel clínico o de otra índole, es importante no solo contar con una serie de normas gubernamentales partiendo del marco legal, sino que también es necesario contar con compendios éticos que deben de acompañar el cabal desarrollo de cualquier investigación y que realmente les compete a los comités de ética en investigación (CEI), los cuales se ocupan de hacer una revisión exhaustiva de todo el proceso desarrollado, partiendo no solo de la metodología utilizada, sino también de la revisión documental y sobre todo de los fundamentos de los postulados éticos utilizados a nivel de la investigación. (Suárez Obando et al., 2018, p. 306).

Estos comités "se han ido posicionando en las instituciones universitarias, pero aún hoy, están demandando mayor presencia, visibilidad y divulgación de su acción, funciones e incidencia en el marco científico-investigativo y social” (Sánchez Sánchez, 2011, p. 426). Especialmente en áreas como las ciencias sociales, las ingenierías, las ciencias básicas, las artes y las humanidades.

Dentro de las funciones de estos organismos, entre otras, se encuentra el de servir de control previo de las investigaciones o publicaciones para evitar o prevenir las posibles afectaciones, así como los conflictos de interés que se puedan generar, además estas entidades deben supervisar el 
desarrollo de las investigaciones y en el caso de que se encuentren afectaciones o posibles daños o incumplimientos de la normatividad, los comités tienen la potestad de interrumpir dichos procesos para evitar que se generen o se extiendan los perjuicios.

Los Comités han tenido más aplicación en el ámbito biomédico, pero se están realizado esfuerzos a nivel gubernamental para implementar estos comités en áreas distintas. En las reuniones con expertos que sostuvo en ese entonces COLCIENCIAS (ahora conocido como MINCIENCIAS) para construir unos lineamientos que orienten la revisión de las investigaciones para los comités de ética de la investigación, lo cual derivó en un evento anual que se denominó como: Diálogos Nacionales de Ética en la Investigación que se viene realizando desde el 2013.

Pero realmente, estos esfuerzos se quedan cortos para los procesos de investigación que se están llevando a cabo en el país, es por esto que, en el primer panel relacionado con la pertinencia de los comités de ética en investigación, se llegaron a algunas conclusiones que se plantean a continuación:

La responsabilidad y la honestidad de los comités en investigación debe ser intachable, por consiguiente, en el momento de seleccionar a los miembros y en especial a los directivos, se deben de elegir los más competentes y objetivos posibles, esto, para evitar los prejuicios y mitos que se pueden presentar alrededor de estos comités. Pero adicionalmente, se debe contar con una entidad rectora que regule a dichos comités. Aquí surgen dos posibilidades, la primera contar con directrices del recién conformado Consejo Nacional de Bioética, que como órgano consultivo del Cobierno Nacional, puede incidir para proponer reglas más claras para los CEI; la otra posibilidad es darle mayor fuerza al documento de lineamientos mínimos para los Comités que se está construyendo por las personas que participan en el proceso de implementación de la política de Ética de la Investigación, Bioética e Integridad Científica, la cual se encuentra regulada en la resolución 314 de 2018.

Se están haciendo algunos esfuerzos para mejorar los procesos de conformación de los CEI, aun así no es suficiente, pues las universidades y demás actores del SNCTel, le hacen un Ilamado permanente a Minciencias para continuar generando todo tipo de lineamientos orientados a los investigadores y a los comités de ética.

En la práctica en Colombia, la mayoría de estos comités han tenido las siguientes dificultades: adolecen de respaldo institucional, convirtiéndolo en un ente avalador de proyectos para presentar a convocatorias externas; en muchos casos no han logrado la autonomía suficiente y sus decisiones, lamentablemente, se alinean con los intereses directivos o estratégicos de la institución, y, en muchos casos, restándole importancia a lo ético, no cuentan con el presupuesto y el tiempo suficiente para la formación de sus miembros, ni para cumplir con sus funciones, no se consolidan los procesos por la alta rotación de sus miembros, se nombran personas con pocos conocimiento o inexperiencia en metodología de investigación o en ética de la investigación y no se les capacita; los motivos descritos pueden llevar a revisiones insuficientes de los proyectos y a una falta de verificación de los procesos investigativos (Sánchez, 2011, pp. 429-430), esto se hace evidente en las discusiones que se tienen en los diferentes nodos regionales de Comités de Ética en la Investigación que existe en Colombia, donde la gran mayoría de los miembros esgrimen estas problemáticas de sus propios comités.

En el caso de los CEl que se conforman para evaluar los lineamientos normativos y éticos en las investigaciones con vacunas y medicamentos, los cuales están contemplados en la Resolución 2378 de 2008 del Ministerio de la Protección Social, el problema principal es que su diseño institucional puede presentar dificultades que se verían reflejados en la falta de independencia institucional y posiblemente en problemas de conflicto de interés, esto quiere decir que la forma como se encuentran diseñados, dificulta que sus decisiones prioricen la ética por encima de los intereses de la empresa farmacéutica que los contrata, lo cual se debe a que la contratación es directa; la empresa farmacéutica o el equipo de investigación debe contratar los servicios de la CEl, estos deben solicitar la autorización al INVIMA y cuando la obtienen, proceden con la revisión de los proyectos, pero por el tipo 
de relación que tienen con la empresa que los contrata y buscando relaciones económicas de largo alcance para poderse sostener financieramente, se abre la posibilidad de tomar decisiones a favor de las empresas, desdibujando la ética.

Se aclara que la crítica que se hace en el párrafo a los CEI que se encuentran en el marco de la resolución 2378 de 2008, se hace al esquema o diseño institucional y normativo, no se está denunciando comportamientos inapropiados de comités específicos. Se habla aquí de lo que puede incentivar la aplicación de la norma, esto es, que se prioricen los intereses particulares por sobre las buenas prácticas en la investigación científica.

Por otro lado, la profesora María Florencia Santi, manifiesta en su tesis doctoral sobre "Ética de la investigación en ciencias sociales. Un análisis de la vulnerabilidad en la investigación social”, que existen unos problemas en la ética de la investigación en ciencias sociales, en investigaciones de tipo experimental o etnográfico y en los instrumentos de recolección de información como las entrevistas, las encuestas y los grupos focales como la responsabilidad del investigador en el momento de la recolección de la información a través de diferentes estrategias, que pueden generar riesgos y posibles daños con relación a los participantes, al igual que se puede presentar inconvenientes en el manejo de la confidencialidad de la información brindada y el respeto a la privacidad de esta y la capacidad de identificar la vulnerabilidad de los participantes para proteger su bienestar e interés. (Santi, s.f.).

Los dilemas éticos planteados por la doctora Santi y los riesgos y daños que se pueden generar por estas prácticas, se podrían aminorar si se tuvieran comités de ética en investigación (CEI) suficientemente responsables que pudieran hacer análisis profundo sobre el tipo de investigación a realizar y la metodología a utilizar. Pues los investigadores tendrían mucho más cuidado para desarrollar investigaciones en el área de ciencias sociales y especialmente con comunidades.

Es de advertir que los afectados pueden ir a las instancias judiciales para buscar la reparación de los daños causados por acciones indebidas de los investigadores o para que se apliquen sanciones penales, disciplinarias o administrativas cuando hay daños más gravosos que afecten la salud, integridad física o la vida de los participantes o cuando se cometan otro tipo de delitos o contravenciones, pero a pesar de ello, aún faltan otros mecanismos de control más públicos que eviten conflictos futuros y que generen disuasión de actuaciones inadecuadas por parte de los investigadores, esto es, que atenten contra los derechos de las personas participantes.

La propuesta que se hace aquí es la de contar con un repositorio público, donde los investigadores e instituciones que faltaron a la ética, se encuentren allí durante un tiempo determinado, para que terceros u otros organismos públicos se abstengan a financiar o aliarse, con estas personas o instituciones, durante el tiempo que aparecen en el repositorio. Esto podría servir para que los investigadores y las instituciones puedan mejorar sus acciones en términos investigativos, similar a como sucede con las sanciones que se les aplican a los profesionales por incumplir las normas de carácter disciplinario.

Otro de los temas importantes, tratándose de la ética en la investigación, es el trabajo con comunidades étnicas y campesinas que comparten diversos elementos como lo son: el territorio que habitan, las costumbres, el idioma y en ocasiones los valores.

Estas comunidades comparten una forma de vida, acompañada de una cultura y costumbres que se sustentan en un patrimonio colectivo biocultural, entendida como:

El conocimiento, las innovaciones y las prácticas de los Pueblos Indígenas y las comunidades locales y móviles, mantenidas de manera colectiva e inextricablemente vinculadas a los recursos y territorios tradicionales, a las economías locales, a la diversidad genética, variedades, especies y ecosistemas, los valores culturales y espirituales y las leyes consuetudinarias formuladas dentro del contexto socio-ecológico de las comunidades (Holly Shrumm \& Jonas, 2012). 
Estos grupos sociales cuentan con sus propias reglas y procedimientos que se traducen en protocolos comunitarios (PC), que pretenden regular las diferentes interacciones de la comunidad, en su interior, así como aquellas actividades, incluida la investigación científica, que se desarrollen con el Estado o con terceros, en su territorio o relacionada con: su biodiversidad y con el patrimonio cultural que ha pasado de generación en generación. Precisamente alrededor de estas, se establecen unos derechos consuetudinarios y el reconocimiento de la normatividad nacional e internacional que protegen estos derechos.

En los casos en que otros estamentos desean trabajar con estas comunidades a nivel cultural o territorial se precisa la necesidad de realizar un "Consentimiento Libre, Previo e Informado (CLPI) que involucra procesos en los cuales las comunidades deciden si permitir o no el desarrollo de proyectos que afecten sus tierras o recursos y en qué términos." (Swiderska et al., 2012) Todo ello permite a las comunidades tener un proceso programado a la hora de tomar decisiones relacionadas con las diferentes propuestas o proyectos que se le planteen, al igual que diferentes discusiones y reflexiones relacionadas con los intereses de esta.

El gran riesgo que se corre, es que los actores externos como entidades del gobierno, grupos de investigación o el sector privado con o sin ánimo de lucro, no sigan los términos de los protocolos comunitarios (por desconocimiento o por desinterés) e impongan formas de gestión, proyectos, planes, recolección especies, procedimientos que pueden vulnerar los derechos e intereses de la comunidad, o afectar la sostenibilidad territorial (Swiderska et al., 2012).

Los protocolos son un buen instrumento para establecer las reglas de juego entre las comunidades y los que deseen realizar actividades en sus territorios, como es el caso de muchos investigadores. Con estos se busca proteger los derechos bioculturales de las poblaciones cuando se van a hacer intervenciones territoriales, como es el caso de los procesos de investigación (recolección de especies o trabajar con el conocimiento de las comunidades, etc.), pero en Colombia no se encuentra reconocido jurídicamente de manera explícita, de ahí que se deba instar al gobierno al reconocimiento y protección de los protocolos, hacer un repositorio público donde se pueda encontrar esta información para que los interesados las puedan consultar.

Un ejemplo de Protocolo comunitario en Colombia fue el realizado con la comunidad del Alto San Juan y miembros del ASOCASAN (Asociación Campesina del Alto San Juan), en el año 2010, donde apoyaron instituciones como el Instituto de Investigaciones Ambientales del Pacífico (IIAP), el Programa de Naciones Unidas para el Medio Ambiente (PNUMA) y la organización no gubernamental Natural Justice, donde a pesar de que esta comunidad ha sorteado una serie de procesos para ganar derechos territoriales, aun así, la problemática se continúa centrando en varias preocupaciones relacionadas con los derechos sobre la propiedad colectiva a nivel biocultural del Alto San Juan como lo son:

[...] el uso de los recursos biológicos de sus territorios ancestrales sin consulta previa, a la baja protección del conocimiento tradicional en relación con las plantas medicinales, a la falta de reconocimiento de las prácticas de minería tradicional, al uso insostenible de los bosques, y, a la permanente amenaza de la pérdida de conocimientos y prácticas tradicionales como consecuencia de un crecimiento económico que no contempla prácticas de desarrollo endógeno" (Consejo Comunitario Mayor del Alto San Juan, 2012).

Pero como ya se mencionó, si bien Colombia suscribió el "Convenio sobre Diversidad Biológica - CDB", aún no ha ratificado a través del Congreso de la República el Protocolo de Nagoya sobre el acceso a los recursos genéticos y participación justa y equitativa en los beneficios que se deriven de su utilización, por tal motivo, no existe una normatividad clara que apoye la implementación de los Protocolos Comunitarios. Esta situación y la falta de un control eficiente hace que los intereses 
gubernamentales, del sector privado, de grupos de investigación o de actores que se encuentran al margen de la ley, afecten el desarrollo de las comunidades o que se desconozcan estos protocolos, de ahí que valga la recomendación de "considerar una fase de seguimiento al desarrollo de un PCB, hasta que el tema esté bien posicionado en el gobierno, o quizás comenzar un proceso en toda la región para apoyar estas iniciativas para mejorar su influencia" (López Piedrahita \& Mosquera, 2012, p. 149).

Otra sugerencia que puede servir para el trabajo con comunidades es la posibilidad de que exista un repositorio público, donde los investigadores deban informar el estado de las investigaciones que se hayan hecho o se estén realizando en los territorios, así como el lugar donde se encuentran los resultados publicados, para que los investigadores conozcan qué se ha hecho con las poblaciones y no se repitan investigaciones innecesarias.

\section{Conclusiones}

Si bien aquí se mencionaron algunos aspectos problemáticos de la ética en la investigación, el mismo se distancia de las miradas tradicionales que se enfocan solo en las áreas de la salud y extiende el análisis a temas socio-ambientales, resaltando:

- La importancia de reconocer los límites de los contratos de acceso a recursos genéticos y productos derivados, en donde la participación y el beneficio para las comunidades es limitado, así como la supervisión del cumplimiento de obligaciones por parte de los investigadores.

La necesidad de aprobar el protocolo de Nagoya y darle piso jurídico a los protocolos comunitarios, como mecanismo de las comunidades para formalizar sus normas consuetudinarias y establecer reglas claras para las investigaciones que se quieran hacer en sus territorios o en sus comunidades.

- La importancia para el país de contar con otros mecanismos de control que complementen las acciones de los comités de ética de investigación.

- La necesidad de que la consulta previa y el Consentimiento libre, previo e informado, para investigaciones con comunidades o en territorios comunitarios, se realice no solo para etnias, sino también para las comunidades locales (rurales o urbanas).

La implementación de la política en ética, bioética e integridad científica de Minciencias (antes Colciencias), del cual se espera que pueda comprometer a las instituciones de educación superior, al Estado, a las organizaciones no académicas, a los empresarios y a los investigadores y se alcance en el mediano plazo, una cultura de la ética en la investigación en Colombia.

Dentro de las cuestiones que quedaron abiertas (es decir, que no se mencionan en este texto) y que pueden servir de líneas adicionales para indagar y profundizar, se encuentran los riesgos en las investigaciones sobre: nanotecnología y nuevos materiales, inteligencia artificial, el manejo de grandes volúmenes de datos, artes, geoingeniería y los asuntos del mar, entre muchos otros.

\section{Referencias bibliográficas}

Abultaif Kadamani, A. (octubre 22 de 2018). Hacia una nueva ética de la investigación en Colombia. Revista Pesquisa Javeriana. https://www.javeriana.edu.co/pesquisa/hacia-una-nueva-etica-de-la-investigacion-en-colombial

Asociación Médica Mundial. (2013). Declaración de Helsinki de la AMM: principios éticos para las investigaciones médicas en seres humanos. Obtenido de sitio web de Asociación Médica Mundial: https://www. wma.net/es/policies-post/declaracion-de-helsinki-de-la-amm-principios-eticos-para-las-investigaciones-medicas-en-seres-humanos/

Consejo Comunitario Mayor del Alto San Juan. (2012). Protocolo Comunitario Biocultural para el Territorio del Consejo Comunitario Mayor del Alto San Juan ASOCASAN. Tadó, Chocó. Colombia. ASOCASAN. http:// 
WwW.pnuma.org/publicaciones/PCB\%20ASOCASAN_espanol_2012.pdf

Decisión 345: Régimen Común de Protección a los Derechos de los Obtentores de Variedades Vegetales, (1993). https://www.wipo.int/edocs/lexdocs/laws/es/co/coo89es.pdf

Decisión 391: Régimen Común sobre Accesos a los Recursos Genéticos. (1996). https://www.wipo.int/ edocs/lexdocs/laws/es/can/cano11es.pdf

Decisión 486: Régimen Común sobre Propiedad Industrial, (2000). https://www.wipo.int/edocs/lexdocs/ laws/es/can/cano1zes.pdf

Decreto 1320: La consulta previa con las comunidades indígenas y negras para la explotación de los recursos naturales dentro de su territorio., (1998). https://2019.vlex.com/\#search/jurisdiction:CO/ decreto+1320+de+1998/WW/vid/353479122

Decreto 309 de 2000: Se reglamenta la investigación científica sobre diversidad biológica., (2000). https://2019.vlex.com/\#search/jurisdiction:CO/decreto+309+de+2000/WW/vid/43133142

Decreto 3570 de 2011. Por el cual se modifican los objetivos y la estructura del Ministerio de Ambiente y Desarrollo Sostenible y se integra el Sector Administrativo de Ambiente y Desarrollo Sostenible. - VLex. https://2019.vlex.com/\#search/jurisdiction:CO/Ley+3570+de+2011/WW/vid/353524390

Departamento Administrativo de Ciencia, Tecnología e Innovación - Colciencias. (2016). Guía para el reconocimiento y medición de grupos de investigación e investigadores. Departamento Administrativo de Ciencia, Tecnología e Innovación - Colciencias. https://minciencias.gov.co/sites/default/files/ ckeditor_files/guia-reconocimiento-y-medicion-de-grupos-e-Investigadores.pdf

Duque Ortiz, D. (Ed.). (2015). Diálogos Nacionales sobre Ética de la Investigación: Memorias 2013-2014. Departamento Administrativo de Ciencia, Tecnología e Innovación. http://www.unesco.org/new/fileadmin/MULTIMEDIA/FIELD/Montevideo/pdf/Bioet-CNBColombia-Memorias2013-14.pdf

Gómez, D., \& Nemogá, G. (2007). Ilegalidad de la investigación genética en Colombia. Pensamiento Jurídico, o(18). https://revistas.unal.edu.co/index.php/peju/article/view/38610

Holly Shrumm, \& Jonas, H. (Eds.). (2012). Protocolos comunitarios bioculturales: Kit de herramientas para facilitadores comunitarios. Natural Justice. https://naturaljustice.org/wp-content/uploads/2012/05/ BCP-Toolkit-Espanol.pdf

Ley 84: Estatuto Nacional de Protección de los Animales.

Ley número 23 de 1982: Sobre los Derechos de Autor. https://propiedadintelectual.unal.edu.co/fileadmin/ recursos/innovacion/docs/normatividad_pi/ley23_1982.pdf

López Piedrahita, T., \& Mosquera, C. H. (2012). La defensa de nuestro territorio: El protocolo comunitario biocultural de Alto San Juan, Colombia. En Aprendizaje y acción participativos. Biodiversidad y cultura: Exploración de protocolos comunitarios, derechos y consentimiento (pp. 140-150). International Institute for Environment and Development (IIED). https://pubs.iied.org/pdfs/14618SIIED.pdf

Obando, F. S. (2015). Un marco ético amplio para la investigación científica en seres humanos: Más allá de los códigos y las declaraciones. La propuesta de Ezekiel ]. Emanuel. Persona y Bioética, 19(2). https://personaybioetica.unisabana.edu.co/index.php/personaybioetica/article/view/5134

Organización de las Naciones Unidas [ONU] (2011). Protocolo de Nagoya sobre acceso a los recursos genéticosy participación justa y equitativa en los beneficios que se deriven de su utilización al Convenio sobre la Diversidad Biológica. Secretaría del Convenio sobre la Diversidad Biológica. https//www.cbd.int/abs/ doc/prtocol/nagoya-protocol-es.pdf

Osío Uribe, O. (2009). Ronda clínica y epidemiológica La Investigación Clínica y los Comités de Bioética. latreia, 22(4), 407-411.

Pardo Martínez, C. I., \& Cotte Poveda, A. (2018). Indicadores de Ciencia y Tecnología, Colombia 2018 (Indicadores de Ciencia y Tecnología). Observatorio Colombiano de Ciencia y Tecnología. https://ocyt.org. co/Libro2018_Completo/INDICADORES_OCyT_2018\%20Version\%2023-07-19.pdf

Potter, V. R. (1999). Bioethics, biology, and the biosphere: Fragmented ethics and «Bridge bioethics». Hasting Center Report, 29(1), 38-40. Scopus.

Resolución 2378 de 2008: Las Buenas Prácticas Clínicas para las instituciones que conducen investigación con medicamentos en seres humanos. https://www.unisabana.edu.co/fileadmin/Archivos_de_ usuario/Documentos/Documentos_Investigacion/Docs_Comite_Etica/Decreto2378de2008_

ACO.USB./Vol. 20 No. 2 /PP 283-297/julio-diciembre-2020/Medellín, Colombia/ISSN: 16578031 E-ISSN: 2665-3354 
Buenas_practicas_clinicas_unisabana.pdf

Resolución número 1348 de 2014. Por la cual se establecen las actividades que configuran acceso a los recursos genéticos y sus productos derivados para la aplicación de la Decisión Andina 391 de 1996 en Colombia y se toman otras determinaciones—VLex. https://2019.vlex.com/\#search/jurisdiction:CO/la+resoluci\%C3\%B3n+1348+de+2014/WW/vid/526366686

Riquelme U, H. (2004). La medicina bajo el Nazismo: una aproximación histórico-cultural. Tercera parte. Medicina U.P.B., 23(2), 117-141.

Sánchez Sánchez, L. F. (2011). El Comité de Bioética, instancia deliberativa, académica y científica. El Ágora USB, 11(2), 425-432.

Sánchez Vazquez, M. J. (2018). Ética, responsabilidad científica e investigación psicológica. Editorial de la Universidad Nacional de La Plata (Edulp).

Santi, M. F. (s.f.). Ética de la investigación en ciencias sociales. Ginebra, Suiza: Ediciones Clobethics.net 2016.pdf. https://www.academia.edu/29474559/Santi_M._F._\%C3\%89tica_de_la_investigaci\%C3\%B3n_en_ciencias_sociales_Ginebra_Suiza_Ediciones_Globethics.net_2016.pdf

Sentencia C 293, D-3748 de 2002. Corte Constitucional. https://2019.vlex.com/\#search/jurisdiction:CO/ C+293+de+2002/WW/vid/43618347

Siqueira, D., \& Eduardo, ]. (2001). EL PRINCIPIO DE RESPONSABILIDAD DE HANS JONAS. Acta bioethica, 7(2), 277-285. https://doi.org/10.4067/S1726-569X2001000200009

Suárez Obando, F. (2015). A Comprehensive ethical framework for scientific research on human beings: beyond codes and statements: Ezekiel ]. Emanuel's Proposal. Persona y Bioética, 19(2), 182-197. https://doi.org/10.5294/PEBI.2015.19.2.2

Suárez Obando, F., Reynales, H., Urina, M., Camacho, J., \& Viteri, M. (2018). Caracterización de un grupo de comités de ética en investigación en Colombia. Persona y Bioética, 22(2), 303-318. https://doi. org/10.5294/pebi.2018.22.2.8

Swiderska, K., Milligan, Á., Kohli, K., Shrumm, H., Jonas, H., Hiemstra, W., \& Oliva, M. J. (2012). Protocolos comunitarios y consentimiento libre, previo e informado: Ceneralidades y lecciones aprendidas. En Aprendizaje y acción participativos. Biodiversidad y cultura: Exploración de protocolos comunitarios, derechos y consentimiento (pp. 25-42). International Institute for Environment and Development (IIED). https://pubs.iied.org/pdfs/14618SIIED.pdf

Tribunal Internacional de Nüremberg. (1946). El Código de Nüremberg. Centro Interdisciplinario de Estudios en Bioética. http://www.uchile.cl/portal/investigacion/centro-interdisciplinario-de-estudios-en-bioetica/documentos/76028/el-codigo-de-nuremberg

Unesco. (julio 26 de 1999). Conferencia Mundial sobre la Ciencia: Declaración sobre la Ciencia. Declaración sobre la ciencia y el uso del saber científico. http://www.unesco.org/science/wcs/esp/declaracion_s.htm

Nota.

' Este trabajo hace parte del proyecto de investigación: "Análisis del discurso de la Bioética Ambiental en Latinoamérica" que adelanta el grupo CIDPAD en la línea "Pensamiento Ecologizante y formación bioética" del Centro Interdisciplinario de Estudios Humanísticos de la Universidad de San Buenaventura Medellín. 